\title{
The Use of Laue Microdiffraction to Study Small-scale Plasticity
}

\author{
H. Van Swygenhoven and S. Van Petegem
}

Micromechanics is a booming research area experiencing the development of new advanced testing methods at small dimensions. A relatively young but very popular technique involves uniaxial compressing micrometer and sub-micrometer sized objects, usually in the shape of pillars. Research in this field has focused mainly on exploring size effects in single crystal metals. This article demonstrates that Laue microdiffraction allows exploring in-situ the evolving microstructure in the transition regime from elasticity to plasticity, a feature that is not accessible with other techniques but which is essential for the understanding of small-scale plasticity.

\section{INTRODUCTION}

In metals, dislocations, i.e. line defects in the single crystal lattice, are the main carriers of plastic strain. ${ }^{1,2}$ Their motion, multiplication, and annihilation mechanisms contribute to the plastic flow and the strength of the metal. The steady operation and interplay of these mechanisms result in the formation of dislocation entanglements, dislocation forests, and eventually the formation of sub-structures. During the microstructural evolution the metal hardens, which for metals with a face-centered cubic (f.c.c.) symmetry is reflected in a specific multi-stage character of the stress-strain response. ${ }^{3}$ It is known that some of the abovementioned dislocation mechanisms might be restricted when the volume of the crystal is confined. For instance, the well-known dislocation FrankRead nucleation source is believed not to operate anymore in a polycrystalline metal when the grain size is well below $50 \mathrm{~nm}^{.-10}$

The development of the micro-com- pression technique, ${ }^{11-13}$ has allowed deforming single crystal samples with diameters between $200 \mathrm{~nm}$ up to more than $20 \mu \mathrm{m}$, revealing a stress-strain response that is different from the expected multi-stage character. Eyecatching is the enhanced strength for

\section{How would you...}

...describe the overall significance of this paper?

Micromechanics is a booming

5 research area experiencing the development of new advanced testing techniques at small dimensions.

근 Most methods provide information on macroscopic mechanical data. In this paper we demonstrate that in situ Laue microdiffraction is a unique method to follow the evolution of the microstructure during mechanical testing at the scale.

...describe this work to a materials science and engineering professional with no experience in your technical specialty?

It is the ease of dislocation nucleation and propagation and the nature of the dislocation multiplication mechanism that determines the strength of the metallic structure; a concept offering several pathways for strengthening. The microcompression test is a relatively new experimental method that revealed a "smaller is stronger" trend for single crystal micron sized samples. This paper reviews the role of the initial defect structure and the boundary conditions of the testing method on the observed trend by performing micro-focused Laue diffraction.

...describe this work to a layperson?

Here, we discuss a new approach to follow in-situ and nondestructively the evolution of the microstructure of micrometer-scaled objects during deformation. smaller sample sizes (i.e., the so-called "smaller is stronger" trend). This is demonstrated in Figure 1 for the case of nickel single-crystal micropillars. The remarkable strengthening of single crystals in this size range has perplexed materials scientists, because it does not fit into our current understanding of single crystal plasticity theories. The strength (at low strain) of single crystals does not depend strongly on the size but rather on the geometrically predicted dislocation slip system(s) for which the resolved shear stress is the highest, the type of hardening mechanism activated and eventually the initial defect content. An enthusiastic community of metallurgists and material scientists started studying all kinds of pillar sizes and metals, resulting in a lot of publications and a huge interest at international conferences. ${ }^{14-25}$

It has to be recognized that microcompression testing is different from the classical well-lubricated largescale compression experiment where the sample can slide on both sides between the compression platens (DIN $\left.50106^{26}\right)$. One of the major differences in a micro-compression device is that the sample set-up is asymmetric, as is demonstrated in Figure 2: the bottom of the pillar is rigidly fixed to the substrate and the top is a free surface (i.e., one of the platens has infinite large friction; the conditions of the other are rather unknown). Such sample geometry is unavoidable because of the impossibility to align a micrometer-sized pillar between two flat platens. On the other hand, the samples are easy to make when using the focused ion beam (FIB) milling technique.

In the early experiments using the micro-compression technique, it was assumed that the axial compression 
testing configuration provides a stress state over a uniform gauge section of the sample that minimizes stretch or rotational strain gradients, at least at the onset of plasticity and for a suitable aspect ratio of the sample. ${ }^{13,14,27}$ These assumptions resulted from simulations on the testing geometry using an anisotropic, elastic-viscoplastic crystallographic finite-element code. It is, however, well known that the nature of the constraints in a compression test changes the uniformity or heterogeneity of the stress distribution in the sample. These different conditions can lead to important differences in activated deformation mechanisms resulting in differences in developing crystal rotations with the possibility for orientation gradients. The way a sample will deform is determined very early during the loading procedure, and affects the evolution of the dislocation network, which in turn affects the strain hardening behavior.

In a micro-compression experiment the boundary conditions are very difficult to control because it depends not only on the chosen hardware (for instance by the lateral stiffness) but also on many other parameters, which can vary from sample to sample, such as the geometrical outline of sample relative to the indenter, ${ }^{11,28}$ the surface quality of the indenter and the pillar top, and the specific geometry of the sample such as the aspect ratio $^{29}$ and the taper geometry. ${ }^{17,28}$

To improve our current understanding of the boundary conditions of the technique, dedicated experiments and computational simulations have to be performed. The effects of initial crystallographic orientation, sample geometry and friction during single crystal micro-compression deformation has been addressed by Raabe et al. in a crystal plasticity finite element modeling (FEM) study. ${ }^{30}$ The authors demonstrated, among others, that nonzero friction conditions reduce shape instability with a dependence on the initial crystal orientation and that the evolution of crystal orientation scatter during compression is weaker when the friction coefficient is higher. Furthermore they observed pronounced deformation-induced crystal orientation changes including the evolution of substantial orientation gradients taking place at engineering compressive strains of about 0.2. The threedimensional crystal plasticity calculations of Shade et al. ${ }^{31}$ show that the stress distribution in a single crystal pillar with a $\langle 123\rangle$ crystal direction parallel to the compression axis is already non-uniform at $0.3 \%$ strain in the presence of friction. Note that the above-mentioned calculations assume the compression stress is applied on a dislocation-free sample. Using a multiscale dislocation dynamics plasticity (MDDP) approach coupling discrete dislocation dynamics at the microscale with the macroscopic plastic deformation, ${ }^{32}$ it has been shown that in the presence of dislocations the influence of friction can be very different. For instance, starting from the same initial dislocation distribution and density, it was shown that when the upper surface is free to move, one slip system dominates and the deformation is localized in two adjacent slip bands located towards the bottom end of the specimen while the upper portion of the specimen glides freely over these bands and deforms elastically. On the other hand, when the upper end is constrained two slip systems are activated and the localization occurs towards the upper surface of the specimen.

Besides the complexity of the boundary conditions of the micro-compression test, there are other parameters that come into play, such as, for instance, the methodology to produce the single crystal pillars. Most studies have been performed on single crystal pillars that have been synthesized using two different FIB milling methods: the first one called "annular milling" ${ }^{17}$ results in a tapered pillar, whereas the second method, "lathe milling," 13 results in a taperfree pillar. During loading the presence of a taper can obviously induce a stress gradient along the pillar axis, which might have non-negligible effects on the onset of plasticity. Furthermore, it is well known that FIB induces damage to the crystal lattice in terms of defect structures and implanted gallium (the ion usually used for FIB milling). ${ }^{33,34}$ How exactly the induced defects, eventually in the form of a damaged layer, affect the observed plasticity in a single crystal pillar geometry is an open ques- tion. The fact is that it has measurable influences on strength as shown by $\mathrm{E}$. George et al. who demonstrated that molybdenum single-crystal pillars obtained by directional solidification lose their whisker-type characteristics when the pillar diameter is slightly reduced by FIB. ${ }^{34,35}$

The above-mentioned uncertainties, resulting from boundary and alignment conditions of the experimental setup and sample geometry, are at the origin of the often not very reproducible initial shape of the stress-strain curve. It is therefore not possible to determine from the stress-strain curve the onset of plasticity by, for instance, a $0.2 \%$ yield criteria as is done for deformation tests of macroscopic samples (see DIN $\left.50106^{26}\right)$.

Needless to say, to make progress in the understanding of the observed "smaller is stronger" effect in single crystals, there is a need for a measurement technique that allows following the microstructure during deformation and eventually to differentiate between phenomena resulting from boundary conditions of the device or geometrical constraints from the sample, and those phenomena intrinsically resulting from plasticity mechanism in a confined volume.

Laue diffraction is an excellent candidate for such purposes. It is one of the oldest diffraction techniques, which however lost the interest from material scientists because it was only applicable for single crystals. However, with the enhanced performance and focusing conditions at synchrotron light sources, Laue diffraction has gained back in interest, as is witnessed by the papers in the current JOM volume. White beam $\mathrm{x}$-ray Laue diffraction is sensitive to the underlying microstructure and, when performed under load, can reflect the dynamics of the selforganization process of dislocations leading to the formation of sub-grain structures. ${ }^{36}$ Continuous streaking of Laue diffraction peaks can be related to internal strain gradients, while discontinuous streaking evidences the presence of dislocation walls, forming geometrically necessary boundaries. ${ }^{37}$ The path along which a Laue peak moves during deformation can be linked to a specific active lattice plane within the 


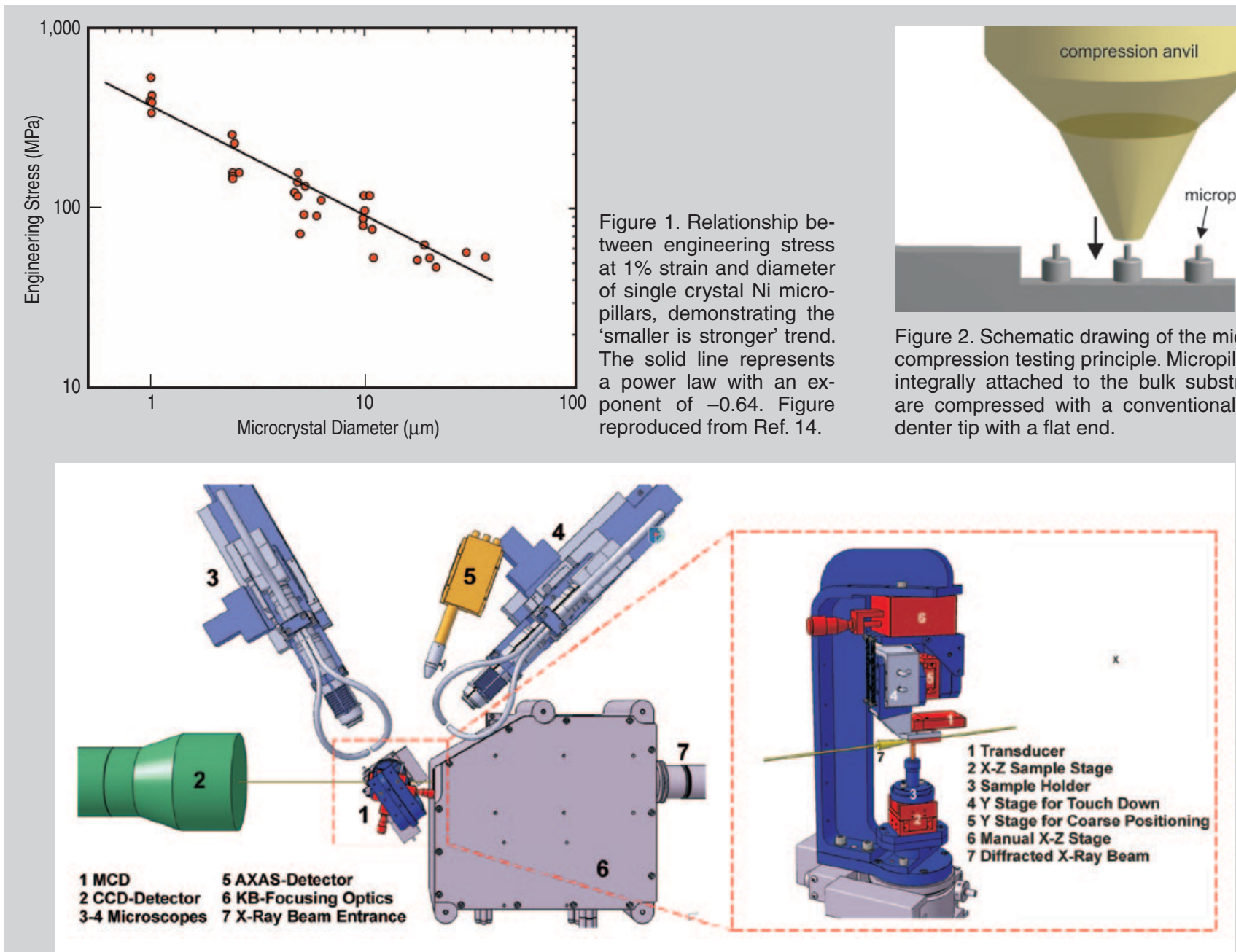

Figure 3. Schematic drawing of the MCD setup at the MicroXAS beam line. The inset shows the micro-compression device in more detail.

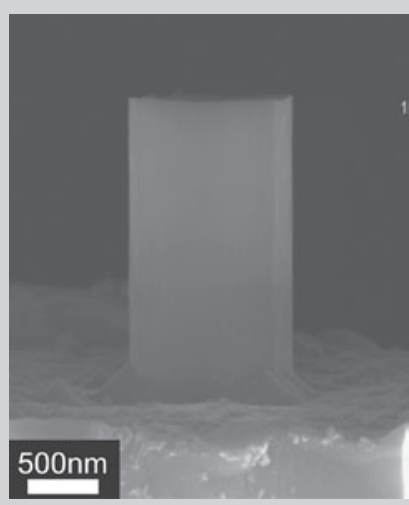

a

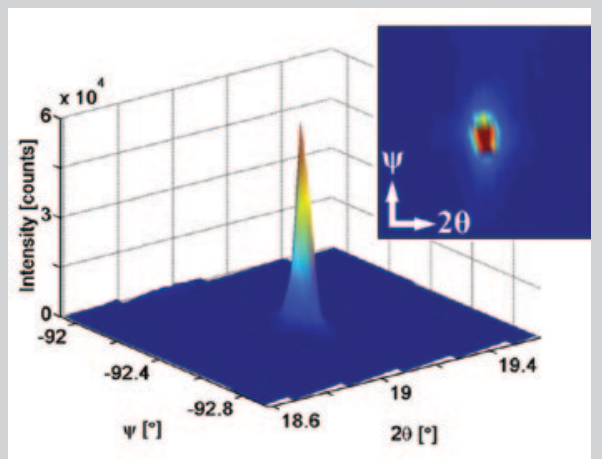

C
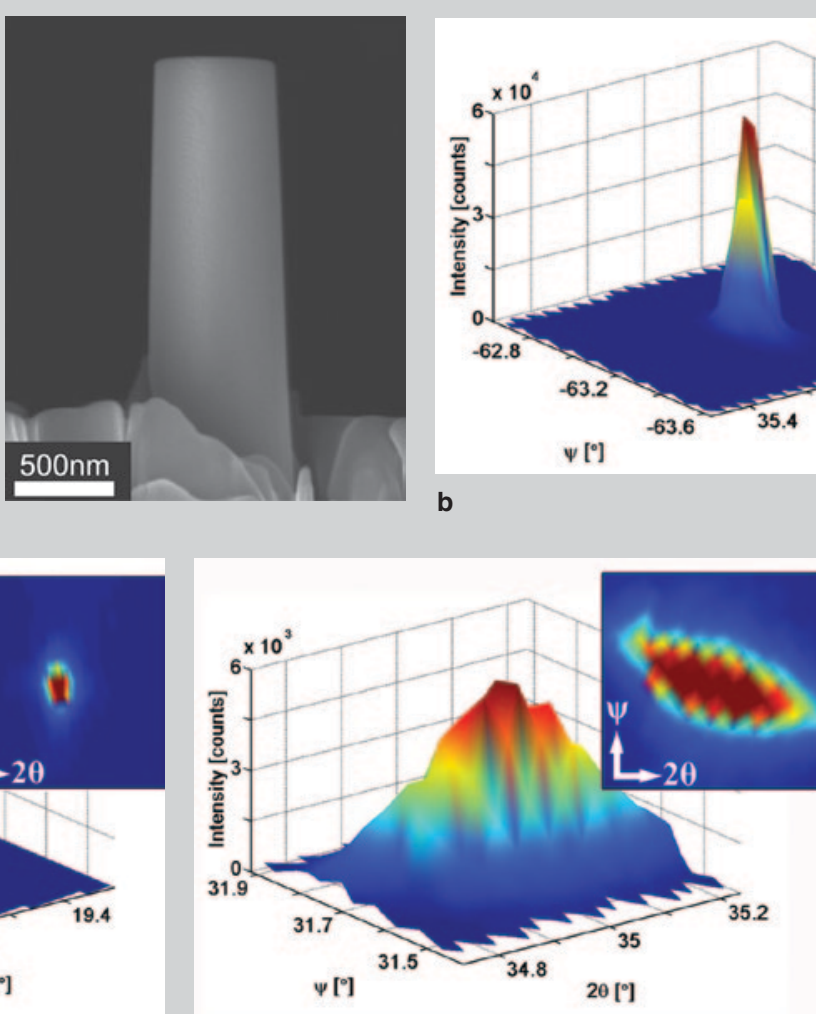

d

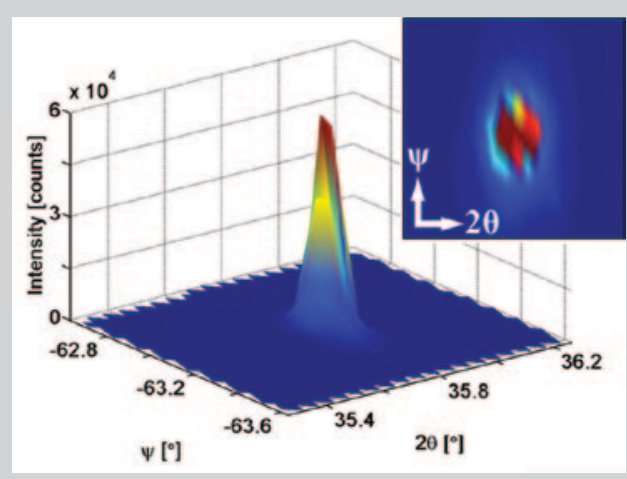

Figure 4. (a) SEM image of a free-standing Mo pillar after etching (left) and after treatment with FIB (right). Representative shapes (3-D and 2-D) of Laue diffraction peaks from (b) a singlecrystal Si wafer (100 $\mu \mathrm{m}$ thick), (c) a DS Mo pillar after etching, and (d) a DS pillar after etching and subsequent FIB treatment. All peaks are plotted in units of radial $(2 \theta)$ and azimuthal $(\psi)$ angles. The radial angle is defined as the angle between the scattering vector and the direct beam, and the azimuthal angle corresponds to the angle between the projection of the scattering vector onto the charge coupled device (CCD) detector plane and the horizontal axis of the CCD plane. In the 2D plots, the dark red color represents the intensity at half maximum. 
rotational Taylor model. ${ }^{38,39}$ From the collective motion of all Laue spots together, the rotation of the local crystallographic orientation can be quantified.

This article highlights selected research results that were obtained using white beam Laue diffraction on single crystal pillars. The examples chosen aim to illustrate the type of information that can be obtained together with their suitability for understanding the effect of the boundary conditions on the outcome of an experiment and for understanding why smaller samples are stronger.

\section{THE SWISS LIGHT SOURCE MICRO-COMPRESSION TEST}

Figure 3 shows a schematic drawing of the in-situ micro-compression setup installed at the MicroXAS beam line of the Swiss Light Source. A white x-ray beam is generated at a conventional undulator source, resulting in an energy distribution ranging from $5 \mathrm{keV}$ to 23 $\mathrm{keV}$. A set of Kirkpatrick-Baez focusing mirrors is used to focus the beam down to a size less than $1 \mu \mathrm{m}$ in the focal plane. The compression experiments are performed with a custom designed micro-compression device (MCD), shown in detail in the inset of Figure 3. It consists of a set of 11
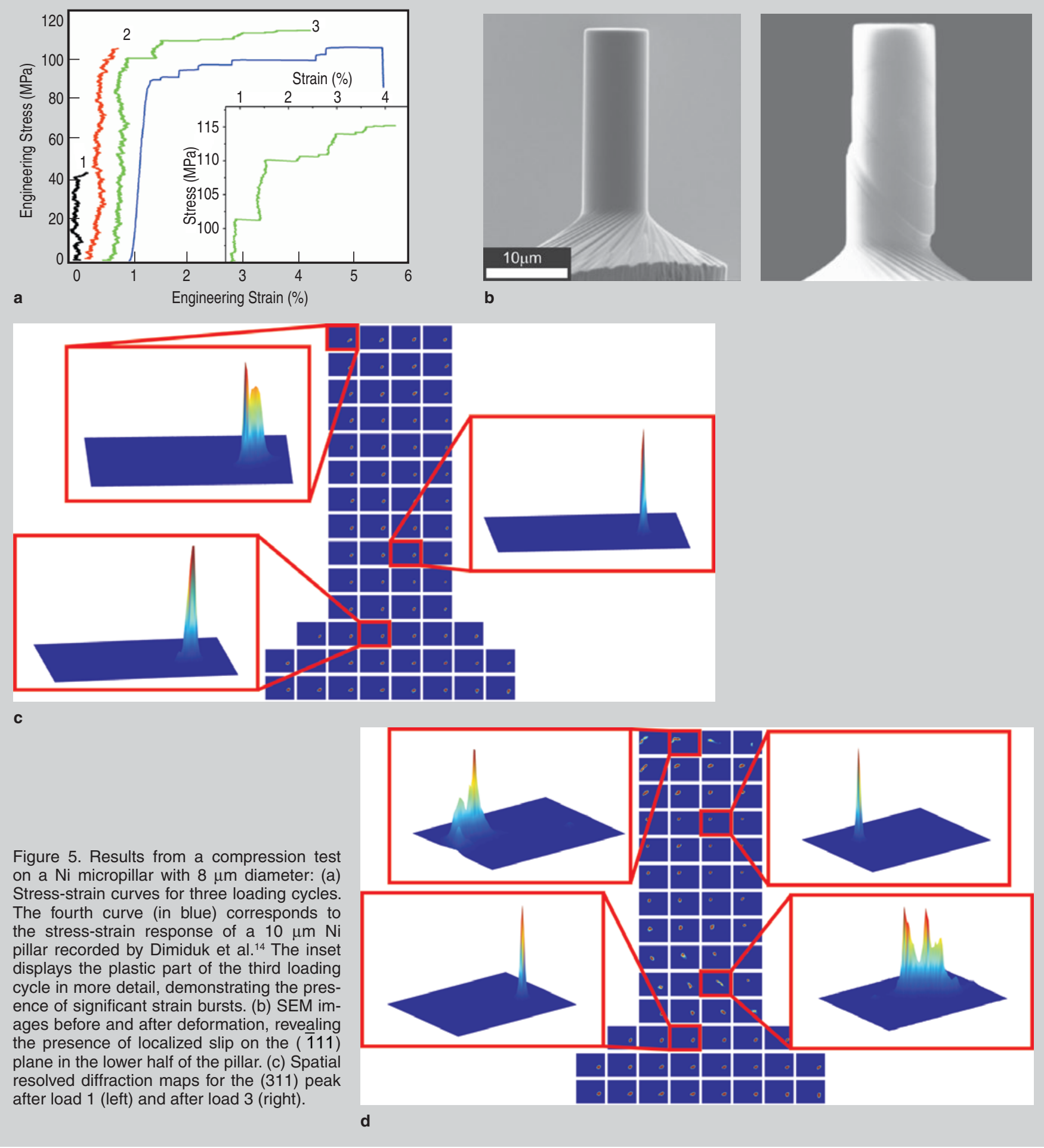

Figure 5. Results from a compression test on a Ni micropillar with $8 \mu \mathrm{m}$ diameter: (a) Stress-strain curves for three loading cycles. The fourth curve (in blue) corresponds to the stress-strain response of a $10 \mu \mathrm{m} \mathrm{Ni}$ pillar recorded by Dimiduk et al. ${ }^{14}$ The inset displays the plastic part of the third loading cycle in more detail, demonstrating the presence of significant strain bursts. (b) SEM images before and after deformation, revealing the presence of localized slip on the ( $\overline{111})$ plane in the lower half of the pillar. (c) Spatial resolved diffraction maps for the (311) peak after load 1 (left) and after load 3 (right). 
linear stages, two tilting stages, and one rotary positioner, which are used for precise alignment of the micropillars with the compression anvil and with the x-ray microbeam. The actual compression test is performed with a standard one-dimensional Triboscope transducer from Hysitron, Inc. The diffracted x-ray beam is detected by a charged couple device (CCD) detector positioned in a Laue transmission geometry at typical sample-to-detector distances of $\sim 50 \mathrm{~mm}$. Emitted fluorescence light from the sample is detected by a single element analogue x-ray acquisition system (AXAS), allowing the reconstruction of the sample position in laboratory coordinates. This method unambiguously determines the sample position relative to the micro-focused $\mathrm{x}$-ray beam. Two high resolution microscopes are used to monitor the alignment in two perpendicular planes during the compression anvil approach and the actual compression experiment.

To check the stiffness of the machine, calibration indents with a standard Berkovich indentation tip were performed on quartz resulting in an indentation modulus of $76 \mathrm{GPa}$, in reasonable agreement with reported literature values. ${ }^{40}$ It has to be mentioned that displacement measurements with a transducer are very sensitive to thermal drift and that the in-situ measurements are not executed in a controlled environment. Indentation software programs usually allow for automatic correction for such a drift, which, unfortunately, cannot be applied for the long in-situ measurements. Further details on the experimental set-up can be found in the online material of Reference 41 .

\section{INITIAL MICROSTRUCTURE OF PILLARS}

There exists clear evidence that the plastic response and the strength of micrometer-sized single crystal pillars may be quite sensitive to the initial defect content. This was recently demonstrated for the case of molybdenum pillars that are obtained after etching away the surrounding $\mathrm{NiAl}$ matrix in a directionally solidified (DS) NiAl-Mo eutectic grown composite. The DS pillars were found to yield at stresses close to the theoretical strength independent of pillar size (in the range 350 1,000 $\mathrm{nm}),{ }^{42}$ reminiscent of whisker behavior. ${ }^{43}$ When the DS pillars were slightly pre-strained before etching and subsequent compression, their strengths decreased dramatically. Furthermore the stress-strain curves became stochastic and exhibited moderate size dependence. ${ }^{44}$ Interestingly, a substantial decrease in the strengths of the DS pillars was also observed after FIB milling. ${ }^{35}$

Therefore, the characterization of the initial microstructure is an important first step in understanding the observed mechanical properties of micropillars. This can be done using transmission electron microscopy. But for this purpose, thin film lamellae have to be cut out of the pillars, where the use to FIB cannot be avoided (see for instance Reference 45). On the other hand, $x$ rays interact weakly with matter and are therefore well suited to investigate the microstructure of entire micrometer-sized specimens. When working in transmission geometry the pillars have to be freestanding (i.e., a free pathway for the incoming and diffracted beam), which is a tedious task in case of the DS molybdenum pillars, as they are only separated by a few micrometers. The technique used to obtain a freestanding pillar out of a forest of pillars has been described in Reference 46. As an alternative, a differential aperture technique can be used to study the microstructure of non-free standing molybdenum pillars. ${ }^{47}$

Figure 4a shows scanning electron microscopy (SEM) pictures of a free standing DS molybdenum pillar after etching (left) and after FIB treatment (right). Both pillars have a $\langle 001\rangle$ crystal orientation parallel to the compression axis. Figure $4 \mathrm{~b}$ displays a representative diffraction peak (011) obtained from the as-prepared pillars. For comparison, a (311) diffraction peak obtained from a $100-\mu \mathrm{m}$ thick single-crystal Si wafer measured under the same conditions is shown in Figure 4c. For the molybdenum pillar, the approximate full-widths at halfmaximum (FWHM), defined in units of radial and azimuthal angles, $\Delta 2 \theta$ and $\Delta \psi$, are $0.04^{\circ}$ and $0.05^{\circ}$, respectively. The corresponding values for the silicon wafer are $0.06^{\circ}$ and $0.06^{\circ}$, respectively. In other words, the peak width obtained for the molybdenum pillars is within the resolution of the experimental setup, the latter being determined by the angular divergence of the incident beam and the detector pixel size. ${ }^{46}$

Although it is not known which is the lower detection limit in dislocation density, it can be concluded that the shape of the Laue diffraction peaks observed for DS pillars correspond to a dislocation-poor single crystal pillar. However, if such pillars are treated on the surface with an FIB, using the annual milling procedure, the diffraction peaks start showing intensity streaks. Figure 4d demonstrates this for a (211) diffraction peak. The continuous intensity distribution of the streaked peaks suggests that the deviatoric strain gradients are present not only near the surface, which is directly affected by the gallium ion implantation, but also near the center of the pillar. For the pillar studied, it is not possible to determine if the streaking direction corresponds with an excess dislocation density on a certain slip system, given the large number of possible slip systems in molybdenum and the limited number of diffraction spots obtained. It can however be said that the lengths of the streaks in FIB pillars are usually too large to be explained entirely by elastic strain gradients without the presence of dislocations.

Similar investigations have also been performed on pillars that were FIB synthesized out of a larger single crystal or out of a well annealed large grain embedded in a polycrystalline metal. Moreover, the studies on face-centered cubic pillars suggest an increasing importance of streaking for smaller pillars. ${ }^{48-50}$ Besides the above-mentioned streaking, Laue diffraction has also demonstrated occasionally the presence of lattice misorientations that could be related to planar defects such as twins or low angle grain boundaries. ${ }^{48}$ The origin of such defects is an open question: they might be due to a rearrangement of the statistically stored dislocations under the influence of FIB or they may arise during sample handling prior or after FIB synthesis. Independent on their origin, the presence of planar defects in the pillar body or at the pillar base may induce dislocation pile up effects. To what extent FIB damage will 
influence the mechanical behavior will strongly depend on the initial microstructure. It can be anticipated that FIB damage will have a minor influence on the measured strength for pillars having a microstructure with an internal length scale that is much smaller compared to the pillar size, such as for instance, nanoporous or nanocrystalline pillars. ${ }^{51}$

In summary, Laue diffraction demonstrates that it is not justified to neglect a priori a possible influence of the FIB synthesis procedure on the mechanical properties of small-scale single crystal objects.

\section{EXPLORING THE BOUNDARY CONDITIONS OF MICRO-COMPRESSION}

As mentioned in the introduction, several simulations have been performed to explore the stress distribution upon loading depending on the crystal orientation, the lateral friction, and the sample geometry. ${ }^{30-32}$ In what follows we describe how Laue diffraction can be used as a complementary tool to investigate these important aspects.

Figure 5 shows the compression deformation curves and the Laue maps taken from an $8 \mu \mathrm{m}$ nickel pillar made by M.D. Uchic using the FIB lathe milling technique. The vertical axis for the pillar corresponds closely with a $\langle 123\rangle$ orientation (within $2^{\circ}$ ) and the pillar was found to be free of planar defects prior to deformation. Moreover, as the pillar is rather big, the streaking due to the FIB procedure was found to be negligible. The pillar was deformed in-situ during Laue diffraction ${ }^{52}$ and subjected to three subsequent load-unload cycles. Figure 5a shows the corresponding stress-strain curves together with the stress-strain curve obtained from an exsitu deformation on a $10 \mu \mathrm{m}$ pillar with a $\langle 269\rangle$ crystal orientation (courtesy $M$. Uchic). Comparing the flow stresses of the $8 \mu \mathrm{m}$ in-situ deformed sample and of the $10 \mu \mathrm{m}$ ex-situ deformed sample, the values correspond approximately with what could be expected from the smaller is stronger effect (see also Figure 1). Figure 5b displays an SEM image of the pillar before deformation and after loading cycle 3 . The slip lines indicate significant localized slip in the lower half of the pillar and are in agreement with the slip plane containing the slips systems with the largest Schmid factor. Because of the relatively large size of the pillar compared the x-ray beam, spatial revolved diffraction patterns could be taken before loading and after every loading step (Figures 5c and d). Inspecting the Laue map of the insitu deformed pillar after loading cycle 1 (Figure 5c) clearly evidences the presence of early plasticity at the top of the pillar. On the other hand the lower part of the pillar remains relatively unaffected. A similar Laue map taken after unloading from the third loading cycle (Figure 5d) shows the presence of lattice misorientations at the top and at the bottom of the pillar, in particular in the region of localized slip. Furthermore the upper half of the crystal has rotated relative to the support by approximately $1^{\circ}$. Apart from the region near the top the diffraction peaks in this upper half exhibit mild broadening.

In view of the simulations performed by Akarapu et al., ${ }^{32}$ plasticity that starts at the top may be related to the presence of lateral constraints, an idea further supported by the presence of significant lattice rotations. ${ }^{39}$ Note however, that the upper part of the pillar did move laterally, indicating that the system is far from fully rigid. Furthermore the observed features may also be evoked by a small misorientation between the indenter and the pillar axis. An in-situ investigation on $\mathrm{Au}$ pillars accompanied by crystal plasticity FEM simulations has demonstrated that small misorientations between pillar axis and compression anvil can result in the selection of a slip system that is geometrically not predicted, ${ }^{53}$ as will be discussed in the "in-situ" example below.

In summary, the use of micro-Laue diffraction allows exploration of the details of the microstructural evolution upon applying load, which, together with a computational approach, will allow improvement of our understanding of the boundary conditions of the micro-compression technique in relation to the sample geometry and initial defect content, as well as exploration of whether these conditions eventually contribute to the observed smaller is stronger trend.

\section{IN-SITU LAUE DIFFRACTION DURING DEFORMATION}

In-situ Laue diffraction is an ideal method to study several aspects of the evolving microstructure during loading. For instance, the way the crystal orientation changes can be used as an indication for the activated slip system(s). This allows the study of the applicability of Schmid's law in confined volumes and the role played by initial microstructure on the selection of the slip systems. Other features that can be studied are, for instance, the formation of geometrically necessary boundaries at larger strains and phase transformations. ${ }^{54}$ In what follows this is demonstrated for the case of a gold micropillar.

Figure 6 shows the compression deformation curve and information obtained from the Laue measurements for a $2.2 \mu \mathrm{m}$ gold sample with a compression axis corresponding with a $\langle 123\rangle$ crystallographic axis (i.e., the pillar is oriented for single slip). The geometrically predicted slip system is the ( 111$)$ [101] for which the Schmid factor amounts 0.47. Before loading, the Laue diffraction peaks evidenced a small amount of streaking that, when expressed in terms of excess dislocations $^{36}$ would correspond with a (111) [ $\overline{1} 01]$ slip system. This slip system has a relative low Schmid factor of 0.36 . The orientation of the plane is shown in the inset of Figure 6 a together with the orientation of the ( $\overline{1} 11)$ plane containing the slip system with the highest Schmid factor. Figure 6a shows the stress-strain curve with the corresponding numbers of the Laue diffraction patterns recorded in-situ during deformation. Figure $6 \mathrm{~b}$ displays the shape of the ( $\overline{222}$ ) Laue reflection during the initial raise of the stress-strain curve. Upon loading all recorded Laue peaks broaden in the same direction as the initial streaking direction, additionally the peak position moves. The path (in detector units) followed by the ( $\overline{222}$ ) reflection during deformation is shown in Figure 6c. An arrow marks the peak position prior to deformation. The first movement of each diffraction peak (colored blue) is closely following one line indicated with the arrow, corresponding to the rotation direction 
expected when slip occurs on the (111) plane. Both initial rotations correspond to dislocation activity on a geometrically less favorable slip plane. After loading up to about $55 \mathrm{MPa}$, a rather abrupt change in path of the Laue peak is observed (colored orange), corresponding to dislocation activity predominantly on the classically predicted slip plane (111), which comprises the slip system with the highest SF. Such a behavior was observed for all recorded Laue peaks. Note that SEM observations after deformation indicated the presence of slip lines for both observed slip systems. From the path followed by all Laue spots the rotation of the vertical crystal axis can be derived by means of full Laue pattern fitting, providing details on the development of the rotational gradients during deformation of a single crystal, as is demonstrated in Reference 53.

In summary, the above example demonstrates the existence of two deformation regimes in FIB single-crystal face-centered-cubic pillars. The first one corresponds to the activity on slip systems different from those that are geometrically predicted for uniaxial compression. The second one corresponds with the highest Schmid factor anticipated for ideal uniaxial compression conditions. The first deformation regime is probably to be associated with the boundary conditions of the test. More analysis is, however, needed to pin down the origin of this nonSchmid behavior. For that it would be necessary to investigate the role of initial streaking that is observed in small pillars that are synthesized by FIB, meanwhile not neglecting the role of small misalignments that are unavoidable, even when the test is performed in an SEM. Crystal plasticity finite element simulations performed in the group of Raabe and coworkers on a gold pillar with similar dimensions and the same crystal orientation have demonstrated the importance of small

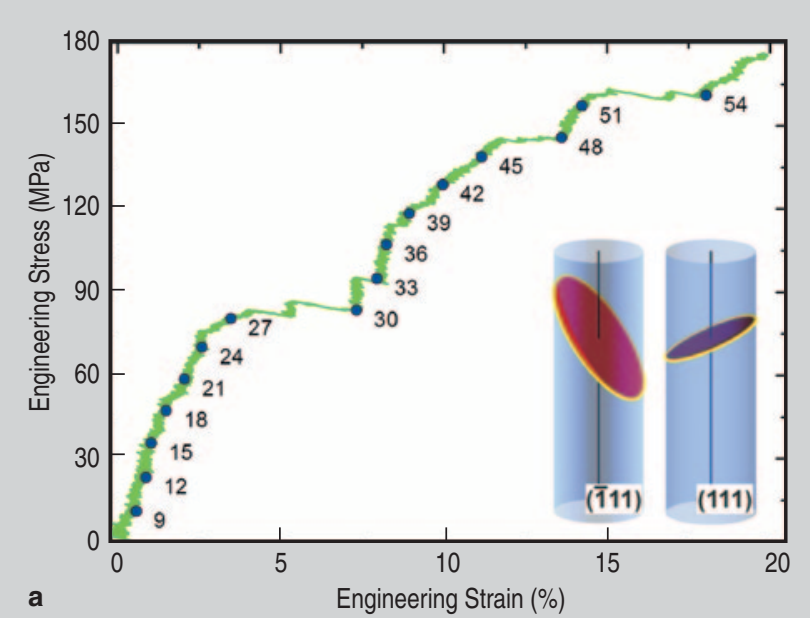

Figure 6. (a) Stress-strain response of a $2.2 \mu \mathrm{m} \quad\langle 123 \tilde{n}$ Au pillar. The numbers of the Laue patterns recorded in-situ during deformation are indicated. The inset displays the orientation of the $(\overline{1} 11)$ and (111) slip planes in the pillar. (b) Two-dimensional representation of the $(\underline{222})$ peak for the indicated Laue pattern number; (c) path followed by the ( 222 ) peak on the detector plane during deformation. The arrows indicate the directions expected for slip on the indicated slip planes. (d) Average shear rates on all 12 slip systems for the compression of micropillars of the same orientation, geometry, and taper as investigated in the current experiments. The boundary conditions were compression at zero friction and tool inclination of $2^{\circ}$ off the ideal punch direction (SF, Schmid factor).

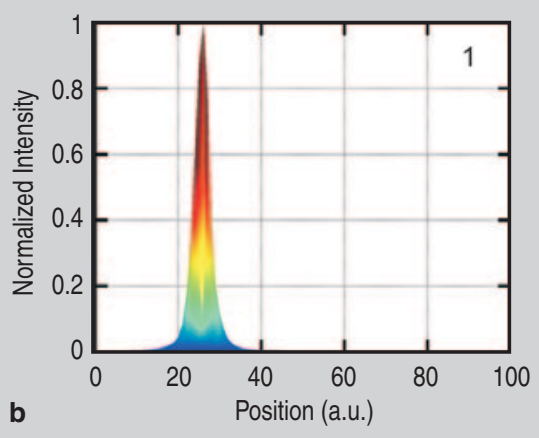

b

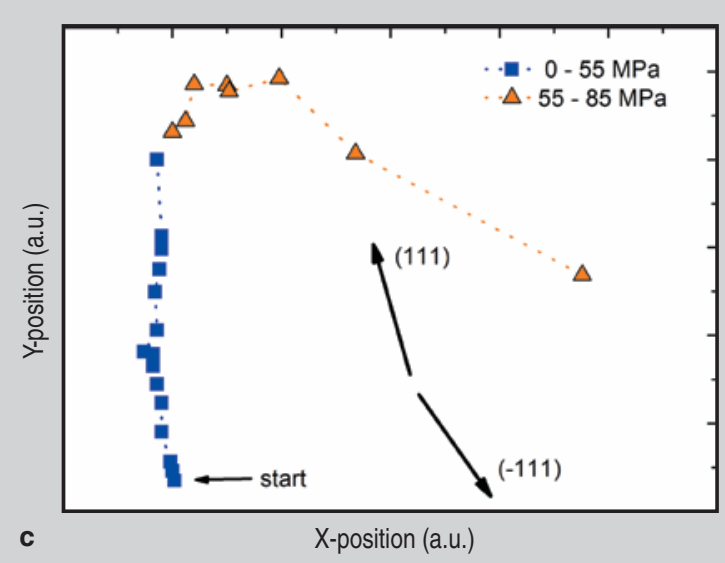

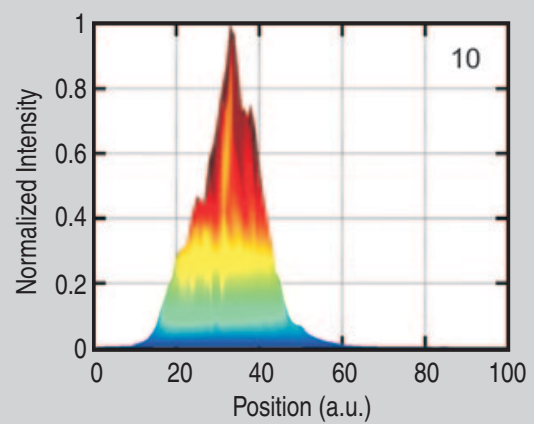

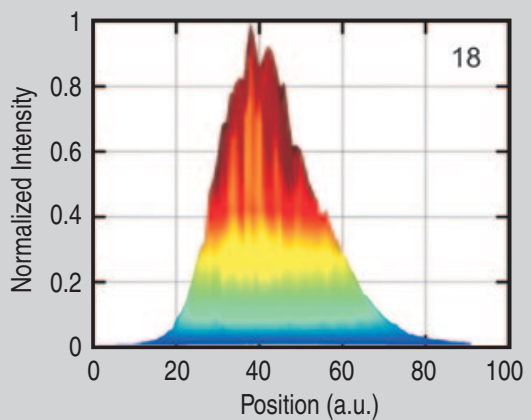

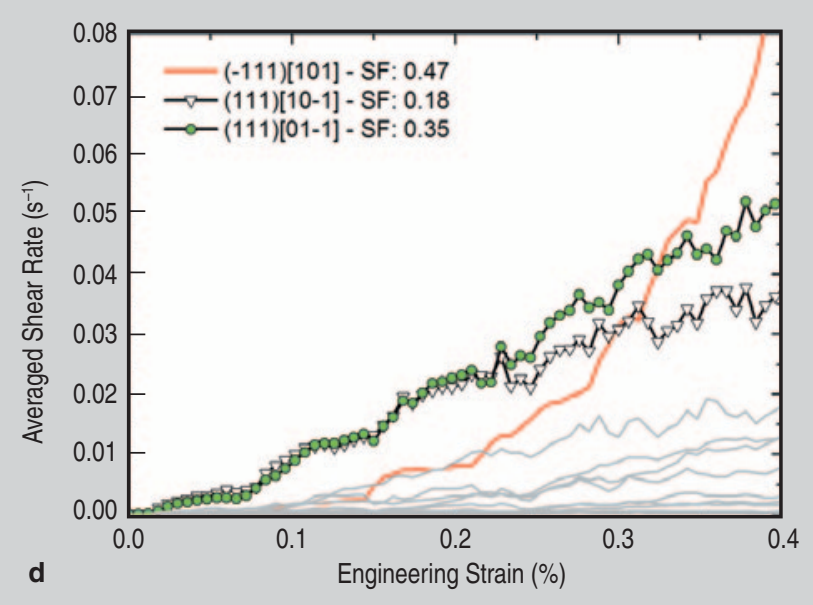


misorientations. ${ }^{53}$ Under a $2^{\circ}$ tool inclination and zero friction, the simulation show first an activity on two slip systems that are geometrically unexpected if a perfect uniaxial compressive stress state is anticipated, before the expected system finally becomes dominant, as is shown in Figure 6d. Beyond 0.3\% strain the geometrically predicted slip system reaches the highest shear rate.

\section{CONCLUSIONS}

The above examples demonstrate that in-situ Laue microdiffraction is an excellent tool to investigate some of the open questions related to microcompression and small scale testing in general. Laue diffraction can give information on the initial microstructure in some of the samples, the influence of FIB synthesis method on the deformation behavior of pillars with diameters below $5 \mu \mathrm{m}$, the influence of friction on the activated slip systems, the effect of initial misorientations on the outcome of a compression test, etc. When combined with computational methods such as those in References 30-32 it will provide the correct understanding of the boundary conditions of the micro-compression test.

But there is an even brighter future for Laue microdiffraction in combination with micro-compression. Laue diffraction has the potential to answer questions of crucial importance related to the observed smaller-is-stronger trend. It will allow differentiating what comes from nucleation aspects ${ }^{16}$ and what comes from hardening aspects. ${ }^{53}$ Indeed, since Laue diffraction allows following the different activated slip systems, it has potential to provide additional details on the size dependent hardening in single crystals. Again, to achieve such a goal, experiments will have to be accompanied by simulations. In particular, the combination with discrete dislocation plasticity methods is very promising. ${ }^{55-59}$

\section{ACKNOWLEDGEMENTS}

The authors gratefully acknowledge the great support of the MicroXAS beamline crew: Dr. D. Grolimund, Dr. C. Borca, and M. Willimann. Thanks go also to the collaborating groups of Dr. E.P. George, Dr. M.D. Uchic, and Dr. C.A. Volkert who provided pillars discussed in this paper and to the Ph.D. students of the Material Science and Simulation group, R. Maaß, J. Zimmermann, C. Marichal, M. Weisser, and S. Pierret.

\section{References}

1. D. Hull and D.J. Bacon, Introduction to Dislocations (Oxford: Butterworth-Heinemann, 2001).

2. J.R. Weertman and J. Weertman, Elementary Dislocation Theory (New York: Oxford University Press, 1992).

3. A.S. Argon, Strengthening Mechanism in Crystal Plasticity, Oxford Series on Materials Modelling (New York: Oxford University Press, 2007).

4. J.R. Weertman, in: Nanostructured Materials; Processing, Properties and Applications, ed. C.C. Koch (Norwich, NY: William Andrews Publishing, 2002), p. 397.

5. E. Arzt, Acta Mater., 46 (1998), pp. 5611-5626.

6. Y. Estrin, H.S. Kim, and F.R.N. Nabarro, Acta Mater., 55 (2007), pp. 6401-6407.

7. H. Van Swygenhoven and J.R. Weertman, Materials Today, 9 (2006), p. 24.

8. H. Van Swygenhoven et al., Phys. Rev. B, 66 (2004), 024101.

9. Z. Budrovic et al., Science, 304 (2004), p. 273.

10. H. Van Swygenhoven et al., Acta Mater., 54 (2006), pp. 1975-1983.

11. M.D. Uchic et al., Materials Research Society Symposium Proceedings, vol. 753, ed. E.P. George et al. (Warrendale, PA: Materials Research Society, 2003), pp. BB1.4.1-BB1.4.6.

12. M.D. Uchic et al., Science, 305 (2004), p. 986.

13. M.D. Uchic and D.A. Dimiduk, Mater. Sci. Eng. A, 400 (2005), p. 268.

14. D.M. Dimiduk et al., Acta Mater., 53 (2005), p. 4065.

15. C. Motz et al., Acta Mater., 53 (2005), p. 4269.

16. J.R. Greer and W.D. Nix, Phys. Rev. B, 73 (2006), 245410.

17. C.A. Volkert and E.T. Lilleodden, Philos. Mag., 86 (2006), p. 5567.

18. D. Kiener et al., Adv. Eng. Mater., 8 (2006), p. 1119. 19. D.M. Dimiduk et al., Science, 312 (2006), p. 1188.

20. C.P. Frick et al., Acta Mater., 55 (2007), p. 3845.

21. D. Kiener et al., Acta Mater., 56 (2008), p. 580.

22. J.R. Greer et al., Mater. Sci. Eng. A, 493 (2008), p. 21.

23. C.P. Frick et al., Mater. Sci. Eng. A, 489 (2008), p. 319 .

24. K.S. Ng and A.H.W. Ngan, Acta Mater., 56 (2008), p. 1712.
25. H. Tang et al., Phys. Rev. Lett., 100 (2008), 185503 26. "DIN 50106 - Testing of Metallic Materials; Compression Test" (Deutsches Institut Für Normung E.V., Burggrafenstraße 6, 10787 Berlin, Germany; 1978).

27. Y.S. Choi et al., Scripta Mater., 57(2007), p. 849. 28. H. Zhang et al., Scripta Mater., 54 (2006), p. 181. 29. D. Kiener et al., Intern. J. Mater. Sci., 98 (2007), pp. 1047-1053.

30. D. Raabe et al., Acta Mater., 55 (2007), pp. 45674583.

31. P.A. Shade et al., Acta Mater., 57 (2009), p. 4580.

32. S. Akarapu et al., Intern. J. Plast., 26 (2010), pp. 239-257.

33. D. Kiener et al., Mater. Sci. Eng. A, 459 (2007), pp. 262-272.

34. H. Bei et al., Appl. Phys. Lett., 91 (2007), 111915. 35. S. Shim et al., Acta Mater., 57 (2009), p. 503.

36. G.E. Ice and R.I. Barabash, in: Dislocation in Solids, Vol. 13, ed. F.R.N. Nabarro and J.P. Hirth (Amsterdam: Elsevier, 2007), Chapter 79.

37. R.I. Barabash, G.E. Ice, and F.J. Walker, J. Appl. Phys., 93 (2003), p. 1457.

38. G.I. Taylor, Proc. R. Soc. Lond. Ser. A-Contain. Pap. Math. Phys. Character, 116 (1927), p. 16.

39. W.F. Hosford, The Mechanics of Crystals and Textured Polycrystals (Oxford, U.K.: Oxford University Press, 1993).

40. W.W. Gerberich et al., J. Mater. Res., 13 (1998), p. 421.

41. R. Maass et al., Phys. Rev. Lett., 99 (2007), 145505. 42. H. Bei et al., Scripta Mater., 57 (2007), p. 397.

43. S.S. Brenner, J. Appl. Phys., 28 (1957), p. 1023.

44. H. Bei et al., Acta Mater., 56 (2008), p. 4762.

45. D.M. Norfleet et al., Acta Mater., 56 (2008), p. 2988.

46. J. Zimmermann et al., Scripta Mater., 62 (2010), pp. 746-749.

47. H. Bei et al., Appl. Phys. Lett., 93 (2008), 071904. 48. R. Maass et al., Scripta Mater., 59 (2008), p. 471.

49. R. Maass et al., Appl. Phys. Lett., 89 (2006), 151905.

50. R. Maass et al., Appl. Phys. Lett., 91 (2007), 131909.

51. S. Van Petegem et al., Nanoletters, 9 (2009), p. 1158.

52. R. Maass, Ph.D. Thesis EPFL 4468, Lausanne, Switzerland (2009).

53. R. Maass et al., Acta Mater., 57 (2009), p. 5996.

54. C.P. Frick et al., Scripta Mater., 62 (2010), p. 492.

55. J. Senger et al., Scripta Mater., 58 (2008), pp. 587-590.

56. J. Senger et al., Phil. Mag., 90 (2010), pp. 617-628. 57. C. Motz et al., Acta Mater., 57 (2009), pp. 17441754.

58. C.R. Weinberger and W. Cai, Proc. Nat. Acad. Sci,, 105 (2009), pp. 14304-14307.

59. J.A. El-Awady et al., Phys. Rev. B, 80 (2009), 104104.

H. Van Swygenhoven, group leader, and S. Van Petegem, senior scientist, are with Material Science and Simulations, ASQ/NUM, Paul Scherrer Institut, CH-5232 Villigen, Switzerland. Prof. Van Swygenhoven is also with the Mechanical Metallurgy Laboratory at Ecole Polytechnique Federale de Lausanne.

\section{S. Van Petegem is a TMS Member!}

To read more about him, turn to page 9. To join TMS, visit www.tms.org/Society/Membership.aspx. 